\title{
Concepciones sobre la producción escrita en académicos que forman profesores básicos
}

\author{
Manuel Rubio M.*
}

\section{Resumen}

El propósito de este artículo es indagar en las concepciones de académicos sobre la producción escrita en la formación inicial docente. Se efectuó un estudio de orden cualitativo sobre la base de entrevistas semi-estructuradas a 10 académicos que imparten diferentes asignaturas, en las cuales la producción escrita juega un rol importante. Los resultados indican que predomina una concepción centrada en la dimensión comunicativa de la escritura, concebida desde los procesos internos que vive el escritor. Si bien dicha concepción se acerca a una orientación de carácter cognitivo muy amplio, no resulta significativa para orientar la producción escrita de los futuros profesores. Por otra parte, la valoración de la escritura que hacen los entrevistados es alta en términos de su desarrollo profesional. No obstante lo anterior, estos no participan en la elaboración de textos relativamente especializados.

Palabras clave: producción escrita, formación inicial docente, concepciones sobre la escritura, prácticas de escritura, alfabetización académica.

\section{Conceptions about the written production of professors training elementary education teachers}

\begin{abstract}
The aim of this paper is to examine professor conceptions about writing production in teacher training programs. A qualitative research was carried out on the basis of 10 interviews to university professors in different disciplines, in which writing production plays a central role. The results indicate that a conception focused on the communicative dimensions of writing, observed from the internal processes of the writer, predominates. Although this conception approaches a very wide cognitive orientation, it is not significantly predominant to guide the future teachers writing production. In any case, the interviewed professors enhance the role of writing in their own professional development; however, they do not participate in the production of relatively specialized texts.
\end{abstract}

Key words: writing production, teacher training, writing conceptions, writing practices, academic literacy.

* Doctor en Lingüística. Academia de Humanismo Cristiano. mrubio@academia.cl 
La formación inicial es uno de los factores que condiciona el quehacer pedagógico de los futuros docentes (Montero, 2002; Borg, 2003). En ella, el estudiante no solo aprende ciertos conceptos y planteamientos relevantes vinculados con las disciplinas que deberá enseñar, sino que también cuestiona, fortalece y/o reconfigura aspectos subjetivos vinculados al contenido curricular escolar.

El futuro docente, a pesar de que haya ingresado con bajos desempeños en aspectos vinculados a la comprensión y producción de textos escritos (Mineduc, 2005b), debe aprender aspectos relevantes de su futura profesión mediante la lectura y escritura de diversos tipos de textos en la mayor parte de las asignaturas que componen su malla curricular.

Desde esta perspectiva, la formación inicial del profesor básico se asienta sobre prácticas letradas heterogéneas y que demandan a los estudiantes conocimiento temático, gramatical, textual y pragmático correspondiente a situaciones de comunicación muy diversas entre sí. Por un lado, debe escribir para aprender en diferentes disciplinas e incluso para construir conocimientos de las experiencias que realiza con otros. Por otro, debe escribir para favorecer el aprendizaje de niños y niñas con intereses, necesidades y entornos culturales diferentes.

Sin lugar a dudas, dichas prácticas se asientan sobre una dimensión que muchas veces no es evidente y que se relaciona con la subjetividad que se comparte en el proceso de formación. En este artículo, basados en el denominado paradigma del pensamiento del profesor (Marcelo, 1987), interesa conocer los significados que le asignan a la producción escrita quienes tienen a su cargo la formación inicial docente. Develar lo que los académicos conocen, creen y piensan, posibilitará comprender, en parte, el entorno cognitivo--emocional en la que los futuros profesores básicos se forman en relación con la competencia comunicativa escrita (Borg, 2003; Woods, 1996).

En concreto, describiremos de manera general las valoraciones, juicios, expectativas, actitudes, etc. de los académicos respecto de la producción escrita y de su propia imagen como escritores y de la imagen que han construido de sus estudiantes como escritores. Esto implica a) caracterizar las conceptualizaciones ligadas al proceso de escribir que posee un grupo de académicos de dos universidades; b) indagar sobre su prácticas de escritura, las valoraciones y eventuales obstáculos que visualizan para la realización de dicha actividad en forma habitual; y c) identificar el rol que le asignan en la formación inicial de los futuros 
docentes, la facilitación que hacen y la percepción que poseen sobre la calidad de los textos escritos que producen sus estudiantes. Cabe indicar que dicha descripción es la primera parte de un trabajo mayor orientado a conocer concepciones que en la formación docente se tienen, tanto por académicos como por los futuros profesores, en relación con la producción escrita.

Es importante destacar que interesa indagar si, en el discurso de un grupo de académicos que forman profesores básicos en dos universidades, existen condiciones para abordar experiencias de producción textual orientadas en tres dimensiones: epistémica, identitaria y pragmática, que son aquellas que se consideran fundamentales en términos de la formación inicial docente (Nonon, 1998).

\section{Marco de referencia}

La producción de textos escritos es un fenómeno complejo. A diferencia del lenguaje oral, la escritura requiere instrucción intencionada para que sea efectivamente adquirida y para que sirva a la realización de diversos propósitos sociales. Esto se explica por el hecho de que la escritura es una tecnología, es decir, un conjunto de conocimientos y habilidades elaborados para manejar algunos problemas de orden práctico. En efecto, la escritura es un artefacto cultural cuyo dominio requiere de esfuerzo consciente y práctica constante (Grabe \& Kaplan, 1996; Casssany, 1999), que cumple, en gran medida, la función de constituirse como un sistema de memoria externa que, a su vez, impacta en el desarrollo de la cognición humana (Pozo, 2001).

Como tecnología, la escritura ha sufrido una serie de transformaciones a lo largo de la historia. Así, además de ser una tecnología manejada por un grupo muy reducido de personas orientadas a conservar el saber socialmente relevante, el manejo de la escritura se ha ido extendiendo a importantes sectores de la población en la mayor parte de los países del mundo y la labor de favorecer su adquisición se le ha asignado como misión a la escuela.

Grabe y Kaplan (1996) plantean que, por la creciente especialización de diversos campos del saber, la escritura se ha ido conceptualizando como un conjunto de destrezas altamente contextualizadas, lo cual significa que un individuo que produce un texto escrito recibe múltiples influencias desde el entorno comunitario y sociocultural en el cual se inscribe y que lo constituyen como escritor. Las prácticas de escritura 
modifican el estatus social, los modos de pensamiento, los conocimientos y la identidad de quienes aprenden a utilizarla.

A grandes rasgos, la producción escrita se puede estudiar desde tres puntos de vista, a saber:

\section{a) El texto producido}

Estos estudios se centran en los recursos lingüísticos y retóricos que posibilitan la construcción textual. En efecto, se pueden analizar los textos producidos para determinar el manejo sintáctico, temático, terminológico y estructural que tienen los escritores. En este sentido, el texto se entiende como un evento comunicativo que demanda cierto grado de esfuerzo cognitivo, se orienta a un propósito y se estructura a base de la relación entre los potenciales interlocutores. Congruentemente, el texto es un punto de entrada a la cognición del sujeto que escribe, al contexto sociocultural en que se inserta, a los principios ideológicos que sustentan su visión de mundo, entre otras dimensiones posibles. Dentro de este contexto, el texto es el resultado de un conjunto de opciones de significación en diferentes niveles, que deja implícita una serie de aspectos para que sean recuperados por los potenciales lectores (Hyland, 2002a; De Beaugrande, 1997; De Beaugrande \& Dressler, 1997, Halliday, 1994; Halliday \& Hasan, 1976; Grabe \& Kaplan, 1996). En esta línea, la enseñanza y evaluación textual en un contexto académico tiende a centrarse en el manejo lingüístico-textual.

\section{b) Los procesos internos que vive el escritor}

Esta perspectiva concibe la producción textual como un proceso, ya sea de profundización en el conocimiento de sí mismo que realiza el escritor o de las operaciones mentales que pone en acción para resolver problemas de orden temático y discursivo. En una perspectiva expresivista, la escritura es un proceso que permite que los escritores encuentren su propia voz para expresarse en forma auténtica y libre, produciendo una prosa fluida, espontánea y de calidad (Grabe \& Kaplan, 1996); su vigencia se fundamenta en un planteamiento crítico frente a la excesiva teorización de los estudios que enfatizan lo social, por lo cual se orientan a una mayor autoconciencia del escritor como sujeto que construye una imagen de sí en el texto que produce (Swearingen, 2002). En una dimensión cognitiva, la escritura se entiende como un proceso de interacción entre el conocimiento previo (lingüístico, textual, pragmático, temático), la problematización de la situación retórica 
(tema, propósito, audiencia) y el control consciente de tareas vinculadas con la pre-escritura, la textualización y la reescritura; en otras palabras, el escritor anticipa, regula y dispone estratégicamente los efectos que busca generar, la imagen que desea presentar de sí, la progresión de las pistas para favorecer la comprensión de los potenciales lectores, la gestión de diversas voces en su propio texto (Hyland, 2002a; Bereiter $\&$ Scardamalia, 1987; Flower \& Hayes, 1981, 1980, 1977; Fabre-Cols, 2004; Boré, 2004).

\section{c) Las prácticas discursivas que constituyen al escritor}

Estos planteamientos, se insertan en el denominado "giro social" que caracteriza a la investigación sobre la producción escrita (Bawarshi, 2003). Dichos estudios destacan las diversas fuerzas sociales que inciden en la cognición del escritor y del propio texto que produce. Desde una línea socio-cognitiva, la propia mente del escritor va cambiando en la medida que este construye nuevos significados, lo cual impacta tanto en su identidad como en las posibilidades de inserción en una comunidad discursiva particular (Flower, 1994). Desde una perspectiva retóricocrítica, se cuestiona la posibilidad de postular procesos universales que caractericen lo que los escritores hacen cuando escriben; el énfasis es concebir la producción escrita como un quehacer público, interpretativo y situado, es decir, contingente, que implicaría la realización de "conjeturas hermenéuticas" en que busca poner en relación las interpretaciones propias con las que tenga una audiencia potencial (Kent, 1999; Olson, 1999). Desde un punto de vista etnográfico, se centra en la descripción de cómo y por qué la gente escribe en condiciones naturales con el fin de comprender como se desarrolla la habilidad de escribir (Grabe \& Kaplan, 1996); así también interesa la relación entre las prácticas letradas del hogar con las de la escuela y entre diferentes escuelas (Schleppegrell, 2004). Desde una postura lingüístico funcional, la escritura se aprende cuando se la utiliza para significar, lo cual implica que el escritor debe irse haciendo consciente del poder de las diferentes elecciones lingüísticas en la construcción de diferentes tipos de significados; concretamente, esto se realiza mediante la descripción de los géneros que se utilizan en el contexto académico, con la finalidad de que los estudiantes puedan reconocer y utilizar sus rasgos léxicos y gramaticales para presentar información de manera precisa, es decir, contar con la habilidad de establecer las opciones discursivas que se esperan según la tarea solicitada (Martin, 1999; Hyland 2002a, Hyland 2002b; Hyland, 2007). 


\section{Metodología}

Se realizó un estudio de casos de orden cualitativo, en el marco del cual se entrevistó a una muestra de 10 académicos que se desempeñan en la formación inicial de profesores básicos en dos universidades: 5 de ellos pertenecientes a una universidad tradicional de la región de Valparaíso y los otros 5 en una universidad privada de la región Metropolitana.

Los sujetos fueron seleccionados considerando el criterio de máxima heterogeneidad posible en relación con su formación profesional, los años de experiencia como académicos universitarios y las disciplinas que enseñan.

En el caso de la universidad pública, tres son profesoras básicas con diferentes especializaciones y otras dos son profesores de enseñanza media. Tres poseen el grado de magíster en educación, una realiza su tesis de magíster en lingüística y dos realizan tesis de doctorado en educación. El promedio de años de experiencia en la formación inicial docente es de 12,5 años. Todas poseen jornada exclusiva en la universidad y enseñan diferentes disciplinas (educación musical, creatividad, formación valórica, metodología de las ciencias naturales, metodología de la investigación, evaluación y talleres de lenguaje y razonamiento).

En el caso de la universidad privada, dos son profesores básicos y tres de enseñanza media. Uno posee el grado de licenciado, tres poseen el grado de magíster, uno se encuentra realizando su tesis doctoral y uno posee el grado de doctor. Todos estos grados en el ámbito de la educación. El promedio de años de experiencia en la formación de docentes es de 17 años. Solo dos poseen jornadas exclusivas. Las disciplinas que enseñan son didáctica de la ciencia, comprensión del medio natural, didáctica de la lengua, didáctica de las matemáticas, evaluación, talleres de práctica y seminario de título.

Se realizó con cada uno de ellos una entrevista individual, guiada por una pauta semi-estructurada, cuyos tópicos fueron: el rol que juegan los textos escritos en el proceso de formación, los conocimientos que los docentes expresan sobre la producción de textos, las creencias en torno a las prácticas de escritura de sus estudiantes. Cada entrevista duró unos 45 minutos.

Para el análisis, se segmentó temáticamente cada entrevista y se implementó un proceso de codificación de tipo inductivo, orientados por procedimientos correspondientes al análisis cualitativo (Strauss \& Corbin, 1990; Valles, 2000). Mediante un proceso sistemático de 
condensación y comparación temática, se identifican coincidencias y disensos entre los entrevistados.

\section{Resultados}

Se ha construido un esquema que sintetiza las concepciones presentes en el discurso de los académicos entrevistados. En el lado izquierdo del esquema, se presenta la conceptualización y funciones atribuidas a la producción de textos escritos. En el derecho, aspectos vinculados a las prácticas de escritura, enfatizando la imagen que dichos académicos han construido de sí mismos como escritores y sus hábitos de escritura.

Figura $\mathrm{N}^{\circ}$ 1: Concepción de los académicos sobre la producción escrita

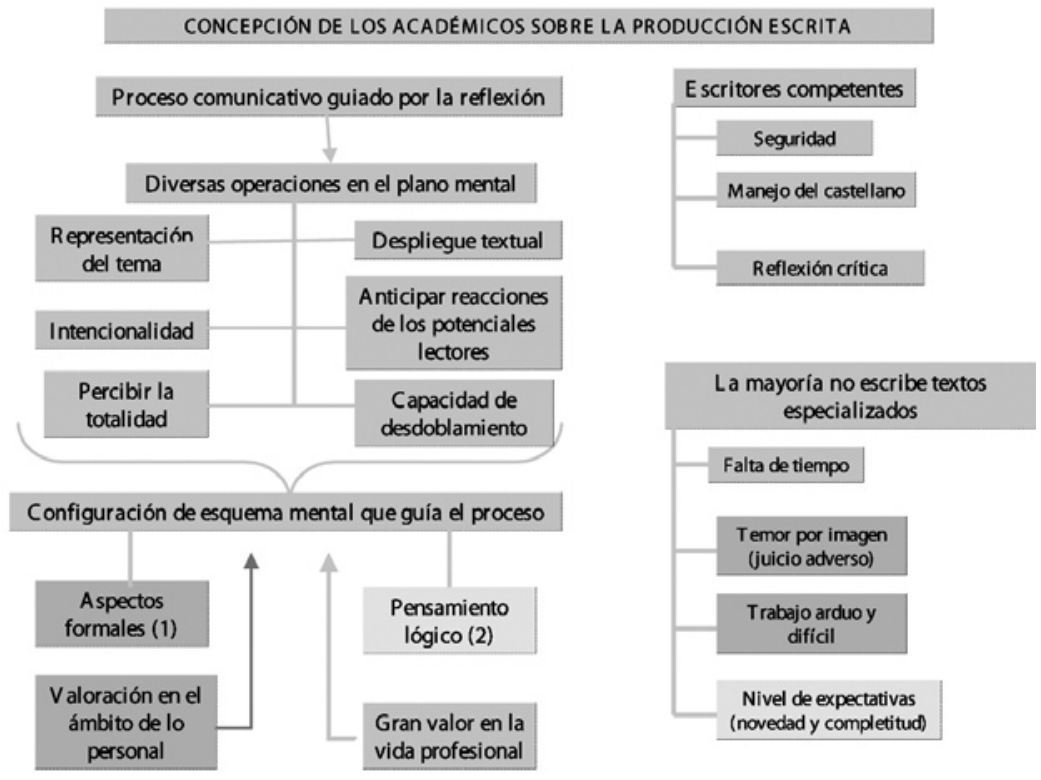

\section{a) Concepciones sobre la escritura}

Los académicos se representan la producción escrita como un proceso comunicativo en el sentido de que supone compartir con otros ideas, conceptualizaciones, puntos de vista e incluso, para algunos, elaborar emociones. En general, dicho proceso es guiado por la reflexión, es decir, una acción intelectual en el que el sujeto razona en torno al contenido de su escrito, sus propósitos y la audiencia potencial. En otras palabras, se concibe como un proceso comunicativo que implica una serie de operaciones mentales que regulan el producto que se desea alcanzar. En 
dicho proceso, los docentes de la universidad pública tienden a poner el acento en aspectos formales y lo valoran desde el ámbito personal y profesional. Por su parte, los académicos de la universidad privada enfatizan el pensamiento lógico y le asignan una alta valoración para la vida profesional.

Cabe entender la descripción efectuada como una síntesis del discurso de los académicos, pues, si bien ellos consideran la producción escrita como un proceso, algunos no explicitan claramente cuáles serían las tareas y operaciones involucradas en él, aunque es posible inferirlas a partir de lo que dicen. Al respecto, destacan en forma manifiesta fundamentalmente la organización y desarrollo de las ideas, y la estructuración del texto. Solamente uno de los entrevistados caracteriza el proceso de escribir en forma explícita y lo centra en la etapa de planificación del texto.

primero, ¿no es cierto?, busquemos qué es lo que pretendemos, anotemos. Ahora, dentro de ese tema, qué planteamientos me parece interesante, ¿no es cierto? Apuntemos eso: en el fondo hagamos el plan de escritura y teniendo el plan de escritura, pongámonos a buscar. Norma al revés que sucede mucho aquí y en muchas partes: primero busco y, después, de lo que encontré, armo lo que me sale, ¿no es cierto? No es así el cuento. El cuento es al revés: cuál es mi propósito, lo pienso, lo describo y luego, ¿no es cierto?, lo separa, hago este plan y, de acuerdo al plan, busco para satisfacer ese plan (A.8).

Esta entrevistada concibe el proceso desde una perspectiva racional viable de ser aplicada a cualquier tipo de texto. Pareciera que es posible pensar solo en un proceso universalmente válido que implica partir por una reflexión en torno a los propósitos y contenidos que se desean exponer. Al parecer, ella se está refiriendo a un tipo de trabajo que supone la consulta a diferentes fuentes. En definitiva, ella pone el acento en la actividad racional de anticipar el quehacer práctico. En su explicación aparece invisibilizada la determinación de la audiencia a la cual potencialmente se destina el texto y el tipo de texto que se desea construir. Cabe indicar, además, que ella cuando explica este proceso solo tiene a la vista un solo tipo de texto y no da evidencia de que otros textos requieran otro tipo de proceso.

En general, los académicos entrevistados no hacen referencias concretas a si el proceso de reflexión y las operaciones mentales que supone 
están condicionadas por el tipo de texto que se produce o por elementos que pudieran obedecer al contexto. Cuando algunos entrevistados de la universidad pública hacen referencia a un condicionamiento de esta naturaleza, se refieren básicamente a la distinción de escribir un texto cuyos destinatarios es el alumnado de básica versus los textos utilizados para exponer un tema como requisito académico.

Los entrevistados de ambas universidades acentúan que un texto escrito es el resultado de un proceso de reflexión ligado al pensamiento lógico y al diálogo. En términos de criterios de calidad, los entrevistados coinciden en la coherencia lógica como propiedad fundamental y la preocupación por facilitar la comprensión de los eventuales lectores. En otras palabras, estarían poniendo el acento en la escritura como una actividad intelectual que exige un ordenamiento global de la información y la capacidad de descentrarse de sí mismos, anticipando las expectativas y condiciones en que se encuentran los potenciales lectores.

Aquellos que trabajan en la universidad pública destacan que sería un registro externo de memoria que debe ceñirse a las normas formales (ortográficas y gramaticales) del castellano y que debe adecuarse a los eventuales destinatarios. En este sentido, ellos reconocen que un futuro profesor se enfrenta a la situación de producir textos para diferentes públicos: sus pares, los directivos, los alumnos de educación básica. Estos entrevistados también hacen referencia a la creatividad para la escritura de ciertos tipos de textos.

Los que trabajan en la universidad privada destacan la actividad intelectual que impone ciertos requisitos de calidad en la expresión de ideas, fundamentalmente en lo que se refiere a la elección de un léxico adecuado. Entre ellos, solo dos destacan la importancia de los aspectos formales (caligráficos, ortográficos y sintácticos).

Cabe indicar que solo dos entrevistadas, pertenecientes a la universidad pública, relacionan la producción escrita con la comunicación de la emotividad y, uno de ellas, la caracteriza como una fuente de deshago y, también, como una herramienta para motivar al alumnado de educación básica.

En síntesis, los entrevistados tienden a poner en relieve aspectos vinculados a la reflexión entendida como una actividad intelectual dirigida a un fin, la cual habría que concebir en términos comunicativos. 


\section{b) Prácticas de escritura}

En la figura $\mathrm{N}^{\circ} 1$, se describe la imagen de escritores que poseen los académicos. Ellos, en general, consideran que son escritores competentes en el sentido de que poseen un importante grado de seguridad para escribir, manejo de un registro culto-formal y la capacidad para reflexionar críticamente sobre un tema y exponerlo por escrito. No obstante, la mayoría no escribe textos especializados en el ámbito pedagógico o de las disciplinas que enseñan. En ambos casos, esto se atribuye a la falta de tiempo. Los académicos de la universidad pública, además, indican que sería un trabajo arduo y difícil. Una de las entrevistadas de esta universidad menciona cierto temor frente al juicio adverso al cual se expone quien escribe. En la universidad privada, aparte de la falta de tiempo, la ausencia del hábito de escribir textos especializados se relacionaría con el nivel de expectativas que los académicos poseen en términos que un texto especializado debe ser novedoso y completo.

Es importante destacar que los académicos de ambas universidades le asignan un alto valor a la producción escrita apelando tanto a su rol en el desarrollo profesional como en el personal. En el campo profesional, serviría como una fuente de reflexión; en el plano personal, como una fuente de auto-conocimiento.

A continuación, detallaremos un poco más esta visión sobre las prácticas de escritura que hemos sintetizado en el esquema.

Los académicos de la universidad pública caracterizan la producción escrita como una actividad que implica un importante grado de complejidad, dado particularmente porque el manejo de ideas supone una serie de dificultades.

Dentro de mis tiempos trato de escribir... Te digo que a mí me cuesta harto. Por eso, te digo que no es simple, porque trato.... A veces me dice la gente igual tienes que tener claro, porque a veces cuando uno es tan formal, como yo que soy tan estructurada, como que tus ideas lógicas.... Entonces lo que trato de hacer es que cuando tengo ideas, es seguir mis ideas y escribirlas en un papel (A.4).

El grado de dificultad que se le asigna al escribir puede estar vinculado al grado de exigencia que los entrevistados se auto-imponen al momento de escribir, el cual consideraría tanto aspectos formales como de contenido. Si bien esta exigencia expresa la valoración asignada a la producción escrita en el ámbito académico, también pudiera estar siendo 
una fuente de inhibición, pues tendrían la noción de que para otros no requeriría un proceso de producción arduo, sino que esta sería más bien una falencia de orden personal.

La valoración positiva de la escritura, como se ha dicho, no significa que los académicos entrevistados escriban habitualmente textos que les permitan exponer sus ideas en circuitos especializados. En el caso de los entrevistados de la universidad pública, son cuatro los que indican que escriben habitualmente. Dos de ellos mencionan que tienen una práctica habitual de escribir textos narrativos; otros dos plantean que producen textos de carácter académico - una de ellas se refiere fundamentalmente a apuntes para los cursos que imparte; la otra a su tesis doctoral.

En el caso de la universidad privada, son cuatro los que se perciben como escritores habituales. Dos de ellos mencionan la edición de apuntes para sus alumnos; otro la realización de resúmenes como un método de estudio y preparación de clases y, otro, la publicación de libros y artículos académicos.

En relación con la falta de hábitos para la publicación de artículos especializados, como hemos dicho anteriormente, la mayoría atribuye tal carencia a problemas de tiempo para una actividad que exige dedicación y esfuerzo. No obstante, algunos de ellos indican otras razones que explicarían tal situación.

Una entrevistada de la universidad pública manifiesta cierta inhibición para escribir este tipo de textos, cuya causa estaría en un cierto temor a enfrentar al grupo de pares.

Publicar una experiencia creo que no tendría ningún problema; con lo que es más vivencial no tengo ningún problema; con lo que es más académico, tengo susto. Me sale bien, pero tengo susto, porque creo que... creo que la academia somos unos grandes descalificadores y que hacemos todo para dar susto

\section{¿Susto?}

Susto.... Lo hago y lo hago bien, pero me da susto, porque estoy convencida de que la academia es una gran inhibidora... para el desarrollo de la escritura (A.5).

En esta perspectiva, la actividad de escribir en el marco de la comunidad académica estaría marcada negativamente, pues el texto escrito se constituiría en un espacio para el despliegue del juicio adverso de los otros. No obstante, esto no se daría con cualquier tipo de texto, sino que 
exclusivamente con el caracterizado como académico, que, en el discurso de esta entrevistada, se opone a lo experiencial. En otras palabras, se podría referir a un texto en que se plasmaría el grado de conocimiento, el nivel de actualización disciplinaria y la reflexión intelectual que un docente efectúa.

Quizás este sea un factor que explica porque algunos entrevistados caracterizan la producción escrita como algo "costoso", "difícil", "demandante". Escribir, en este contexto, implicaría mostrar la actividad intelectual efectuada y la calidad de la misma, es decir, someterse voluntariamente a una evaluación de los pares, un proceso que puede ser fuente de legitimación o atentar contra ella.

Por su parte, en la universidad privada dos son las explicaciones que se articulan en términos de la falta de hábito de escribir textos especializados. La primera es atribuirlo a carencia de tiempo para una actividad que requiere dedicación y esfuerzo. La segunda es el nivel de expectativas que se espera que tenga un texto publicable.

Pero creo que no he sido lo suficientemente responsable como para dejarme el espacio para publicar, no necesario de escribir, sino que para publicar lo que he hecho.... Además, de partida muchas veces me han pedido que vaya a hablar con profesores a otro lado o en un curso o participar en un seminario, y uno escribe lo que allí hace, y lo presenta, pero no lo guarda, porque además también siempre uno siente que le faltó algo, que no quedó completo, que le faltaron cosas. Entonces no tiene mucha gracia publicarlo. Entonces, creo que ese es el gran defecto (A.6.)

De acuerdo con esta entrevistada, no basta que un escrito haya sido validado en instancias académicas, sino que tiene que ser algo "completo", acabado, que indique un manejo profundo del campo disciplinario y algún grado de novedad. Tampoco se toma tales escritos como un texto que puede seguir siendo elaborado. En esta visión, de algún modo subyace una imagen de escritor un tanto minusvalorada, pues en el fondo se cuestiona la calidad de los mismos. Por otra parte, pareciera ser que cuando se piensa en la publicación no se piensa en los profesores que trabajan en el sistema escolar, que fueron los destinatarios a partir de los cuales se articularon, sino que se evalúan desde el punto de vista de una audiencia experta a la cual se desea mostrar una imagen de especialización incuestionable. 
En esta misma línea, la entrevistada señala la necesidad de novedad como un criterio para decidir si un artículo debe o no publicarse, lo cual podría más que ser una fuente de estímulo, transformarse en una suerte de inhibición para cumplir con esta labor.

Pero también como fortaleza, el respeto por lo que hace el otro, o sea, yo admiro a mucha gente que está haciendo cosas en el área didáctica y siempre pienso que ellos ya lo han pensado. Entonces, cuando uno lo pensó, está como ya hecho, escrito y no a renovar (A.6).

Cabe hacer notar que solamente una entrevistada, perteneciente a la universidad pública, indica que el escribir constituye una fuente de desahogo para ella.

Para mí la escritura, básicamente, es un medio de comunicación, un medio de reflexión. Reconozco, además, que es una fuente de desahogo en algunos minutos. Para mí, la escritura es importante... cuando uno escribe.... Por ejemplo, antes de pelear con alguien, escribo; antes de decirles cosas a esas personas, se las escribo; no se las mando, pero se las escribo. Eso ayuda mucho (A.2.).

Esta y otra entrevistada de la misma universidad manifestaron su interés en la producción de narraciones literarias, que, al parecer, constituyen para ellas un menor grado de exposición profesional. Estos textos se configuran con fines pedagógicos, ya sea de motivación del alumnado básico o de su formación en el plano emocional.

Al evaluarse como escritores, los entrevistados de ambas universidades destacan que tienen un buen manejo de la escritura, la capacidad crítica para reflexionar sobre lo que escriben y la seguridad que manifiestan para hacerlo. Esto no significa que para ellos la actividad de escribir esté exenta de esfuerzo. Por el contrario, demanda un tiempo que, en general, consideran limitado.

Ahora bien, esta explicación debiéramos relativizarla considerando, como se indicó anteriormente, las expectativas y temores que inciden en la significación que se configura del escribir en un ambiente académicamente competitivo. En esta línea, debiéramos preguntarnos por cuán amenazante para su imagen académica resulta el exponerse a través del escribir textos especializados. 


\section{c) La producción escrita en la práctica pedagógica}

Un aspecto importante para establecer las concepciones sobre la producción escrita es el rol que se le asigna en el proceso de enseñanza de los futuros profesores básicos. Al respecto, los académicos fundamentalmente aluden a la promoción de la reflexión, pero no indican claramente que intencionen un proceso de producción guiado o que se diferencia según el nivel que cursen los estudiantes. Congruentemente, cuando se refieren a la producción escrita en sus prácticas de enseñanza indican tipos de textos que solicitan y los criterios desde los cuales los evalúan.

En esta línea, según los académicos de ambas universidades los estudiantes muestran deficiencias en exponer las ideas de diversos autores considerando el contexto de producción, es decir, no contextualizarían temporalmente ni disciplinariamente las ideas que seleccionan y exponen.

Al mencionar criterios de evaluación, los académicos entrevistados enfatizan los aspectos formales del texto (ortografía y sintaxis), la legibilidad, la precisión conceptual, el cumplimiento de los objetivos propuestos para la tarea y que el texto producido sea coherente. En la universidad pública, solo una persona menciona la adecuación a los receptores y, otra, la importancia de secuenciar apropiadamente la información. En la universidad privada, los académicos tienden a destacar la referencia a los potenciales lectores.

Algunos académicos de la universidad pública ponen un fuerte énfasis en la caligrafía, dado que el futuro profesor básico debe constituirse en un modelo para sus alumnos en este aspecto. Son cuatro los académicos entrevistados, dos de cada universidad, que considerarían el nivel que cursan los estudiantes como un elemento a tener en cuenta al momento de evaluar.

En síntesis, pareciera ser que aspectos ligados al despliegue temático, a las redes conceptuales que se articulan en el texto y los rasgos estilísticos que contribuyen a manifestar las posturas y la voz del productor no se considerarían en la evaluación de los textos escritos. Tampoco hay referencias a que se intencionen las operaciones mentales mencionadas al caracterizar la producción escrita como un proceso de reflexión. Pareciera que en una parte importante de los entrevistados, importa la consideración a la audiencia, pero dicha consideración es bastante general.

Lo anterior, podría estar indicando que los académicos dan por supuesto que los estudiantes debieran ser capaces de reflexionar en forma 
autónoma, independientemente de que se vayan insertando en un campo disciplinar relativamente especializado.

\section{Conclusiones}

Las representaciones que los académicos de ambas universidades manifiestan en su discurso no difieren sustantivamente. De acuerdo con lo analizado, tiende a predominar una concepción centrada en la dimensión comunicativa de la escritura concebida desde los procesos internos que vive el escritor. En dicha concepción, se destacan operaciones vinculadas con la problematización de la situación retórica y el control del despliegue informativo, lo que permitiría situar a los académicos en una orientación de carácter cognitivo muy amplio, en el cual aparecen invisibilizadas dimensiones relativas a la activación de conocimientos almacenados en la memoria, el condicionamiento que impone el texto que se escribe, la interacción entre diversos subprocesos, la función identitaria y epistémica que tiene la escritura, entre otras.

Si bien, en la línea de los procesos internos del escritor, aparece en el discurso de algunos académicos una orientación más expresivista, esta tiene claramente una importancia marginal. Lo predominante es considerar la escritura como una operación racional fuertemente valorada desde el campo profesional.

No obstante lo anterior, cuando los académicos consideran la actividad de escritura de sus estudiantes, se limitan al producto de dicha actividad, es decir, el texto producido. No se evidencia en su discurso que intencionen procesos de toma de conciencia orientadas a guiar el proceso de producción. El énfasis en su valoración tiende a destacar rasgos de la superficie textual y no se reconoce que haya acciones didácticas orientadas a considerar elementos pragmáticos o que sitúen la escritura como una práctica social. En otras palabras, no aparecen evidencias de que se adopte una perspectiva situada de la producción escrita ni que se promueva la reescritura de los textos facilitando una revisión global del texto.

Por otro lado, pareciera ser que la tarea demandada (y por tanto el tipo de texto que se debe construir) no incide en la descripción del proceso. En este sentido, se concibe la escritura como una habilidad general que invariablemente implica las mismas operaciones. No se consideran aspectos vinculados a las demandas que implica iniciarse en un campo temático relativamente especializado y a los rasgos genéricos que tales campos implican. 
Los académicos de ambas universidades tienen una imagen de sí mismos como escritores competentes, es decir, que manifiestan seguridad en sus habilidades y en su manejo del castellano para la construcción de textos y para reflexionar sobre estos desde una perspectiva crítica. Sin embargo, salvo unos pocos casos, los académicos entrevistados no participan en la elaboración de textos especializados. Si bien consideran que es relevante en la vida académica, no lo hacen y atribuyen este hecho a la falta de tiempo y a la auto-imagen que se desea validar como expertos.

De acuerdo con los resultados expuestos, surge la necesidad de indagar en las prácticas de escritura que los académicos promueven en el alumnado y en la percepción que han ido configurando respecto de la incidencia de la comunidad académica en la facilitación u obstaculización de la producción de textos que trasciendan la docencia y la función motivacional de alumnos de educación básica. En este sentido, sería interesante indagar más profundamente en la propia imagen de escritor que los académicos han formulado de sí mismos.

Al mismo tiempo, resulta conveniente indagar sobre el rol que le asignan los académicos a la experiencia pedagógica como una fuente de conocimientos viable de ser sistematizada y comunicada en circuitos especializados, pues, hipotetizamos, que habría una visión que sitúa las prácticas de escritura en un alto nivel de experticia que deslegitima los potenciales aportes que pudieran realizar.

\section{Bibliografía}

Bawarshi, A. (2003). Genre and the invention of the writer. Reconsidering the place of invention in composition. Logan, Utah: Utah State University Press.

Bereiter, C. \& Scardamalia, M. (1987). The psychology of written composition. Hellsdle, N. Jersey: LEA.

Boré, C. (2004). "Contribution des brouillons á la connaissance de l'écritures scolaire". Le François Aujourd'hui 144, 42-51.

Borg, S. (2003). "Teacher cognition in language teaching: A review of research on what language teacher think, know, believe, and do". Language Teacher 36, 81-109.

Cassany, D. (1999). Construir la escritura. Barcelona: Paidós. 
De Beaugrande, R. (1997). New foundations for a science of text and discourse. Cognition, communication, and the access to knowledge and society. New York: Academic Press.

\& Dressler, W. (1997). Introducción a la lingüística del texto. Barcelona: Ariel.

Fabre-Colls, C. (2004). "Les brouillons et l'école ce qu'a change la critique génétique”. Le François Aujourd'hui 144, 18-24.

Flower, L. (1994). The construction of negotiated meaning. A social cognitive theory of writing. Carbondale: Southern Illinois University Press.

\& Hayes, J. (1977). "Problem-solving strategies and the writing process”. College English 39 (4), 449-461.

(1980). "The cognition of discovery: defining a rhetorical problem". College Composition and Communication 31, 21-32.

(1981). "A cognitive process theory of writing". College composition and Communication 32(4), 928-950.

Grabe, W. \& Kaplan, R. (1996). Theory and practice of writing. New York: Longman.

Halliday, M. A. K. (1994). An introduction to functional grammar. London: Arnold.

\& Hasan, R. (1976). Cohesion in English. London: Longman.

Hyland, K. (2002a). Teaching and researching writing. Essex: Pearson Education

(2002b). "Genre: language, context, and literacy". Annual Review of Applied Linguistics, 22, 113-135.

(2007). "Genre pedagogy: language, literacy and L2 writing instruction". Journal of Second Language Writing, 16, 148-164.

Kent, T. (1999). Introduction. En Kent, T. (ed.) Post-process theory, beyond the writing-process paradigm (pp. 1-6). Illinois: Southern Illinois University.

Marcelo, C. (1987). El pensamiento del professor. Barcelona: Ediciones CEAC. 
Martin, J. (1999). "Mentoring semiogenesis: 'genre-based' literacy pedagogy". En F. Christie (ed.) Pedagogy and the shaping of consciousness. London: Continuum, 123-154.

Mineduc (2005). Informe de la Comisión sobre Formación Inicial Docente. Santiago: Mineduc.

Montero, L. (2002). "La formación inicial, ipuerta de entrada al desarrollo profesional?" Educar 30, 69-89.

Nonnon, E. (1998). "Quelle transposition des theories du texte en formation des enseignants?" Pratiques 97-98, 153-170.

Olson, G. (1999). "Toward a post-process composition: abandoning the rhetoric of assertion". En Kent, T. (ed.) Post-process theory, beyond the writing-process paradigm (pp. 7-15). Illinois: Southern Illinois University.

Pozo, J. I. (2001). Humana mente. El mundo, la conciencia y la carne. Madrid: Ediciones Morata.

Schleppegrell, M. (2004). The language of schooling. A functional linguistics perspective. Mahwah, N. J.: Lawrence Erlbaum Associates Publishers.

Strauss, A. \& Corbin, J. (1990). Basics of qualitative research. Gounded Theory, procedures and techniques. California: Sage Publications.

Swearingen, C. J. (2002). "Rhetoric and composition as a coherent intellectual discipline: a meditation". En G. Olson (ed.), Rhetoric and composition as intellectual work (pp. 12-22). Carondale and Edwardsville: Southern Illinois University Press.

Valles, M. (2000). Técnicas cualitativas de investigación social. Madrid: Síntesis.

Woods, D. (1996). Teacher cognition in language teaching. Beliefs, decisionmaking and classroom practice. Cambridge: Cambridge University Press. 